\title{
El gasto de bolsillo en salud: el caso de Chile, 1997 y 2007
}

\author{
Camilo Cid Pedraza ${ }^{1}$ y Lorena Prieto Toledo ${ }^{2}$
}

Forma de citar Cid Pedraza C, Prieto Toledo L. El gasto de bolsillo en salud: el caso de Chile, 1997 y 2007. Rev Panam Salud Publica. 2012;31(4)310-16.

RESUMEN Objetivo. Determinar el impacto, las características y los cambios del gasto de bolsillo en salud de los hogares en Chile entre 1997 y 2007.

Métodos. Estudio descriptivo y econométrico basado en encuestas de hogares con información transversal sobre gasto en dos años -1997 y 2007- para el Gran Santiago. Se revisa la evolución de indicadores del gasto por quintiles de gasto per cápita del hogar. Se utiliza la metodología propuesta por la Organización Mundial de la Salud para el análisis econométrico de los determinantes del gasto de bolsillo y del gasto catastrófico. También se calculan índices Gini para el análisis de equidad.

Resultados. El gasto de bolsillo en salud de los hogares del Gran Santiago aumentó significativamente (39,5\% per cápita). La razón de gasto en salud del quintil 5 respecto al 1 disminuyó, pero en razón de que el aumento fue menor en el quintil 5. El gasto de bolsillo en 2007 sigue estando determinado por la presencia de grupos de riesgo: menores de 5 años de edad $y$, aunque en menor medida, mayores a 65 años. El gasto catastrófico disminuyó levemente y la presencia de adultos mayores persiste en aumentar dicho riesgo. La presencia de mujeres en edad fértil tiende a ser un atenuador del gasto de bolsillo.

Conclusiones. El gasto de bolsillo en salud de los hogares ha crecido y es alto, y su influencia en la desigualdad sigue siendo significativo. Se detectan efectos de programas como el AUGE en la contención del gasto de bolsillo, pero se necesitan nuevas políticas de protección financiera que ataquen el problema.

Palabras clave Financiación de la salud; gastos en salud; financiación personal; equidad en salud; Chile.

En prácticamente todos los países, las políticas de salud en el ámbito del financiamiento apuntan a la protección financiera de las personas y familias. Por lo tanto, se concentran en generar una disminución del gasto de bolsillo de los hogares que se destina a la atención de salud, por ser esta la forma de financia-

Pontificia Universidad Católica de Chile, Departamento de Salud Pública, Santiago, Chile. La correspondencia se debe dirigir a Camilo Cid Pedraza, ccid@med.puc.cl

2 Escuela de Administración de Negocios para Graduados (ESAN), Lima, Perú. miento de la salud considerada menos justa y menos eficiente $(1,2)$. Además, desde el punto de vista de las políticas de salud, preocupa que el gasto de las familias en salud no se vuelva un evento catastrófico ni sea un factor que las lleve a caer o permanecer en la pobreza $(1,2)$.

En Chile, el sistema de seguridad social en salud está compuesto por el Fondo Nacional de Salud (FONASA) y las Instituciones de Salud Previsional (ISAPREs), con una prima obligatoria de 7\% del salario por trabajador para cubrirlo a él y a su familia. En el sistema de
ISAPREs la prima está asociada a riesgos y a la cantidad de beneficiarios del plan contratado. De esta manera, 7\% opera como un piso para la prima y se complementa con una "prima voluntaria" ajustada al costo esperado. En 2009 la prima promedio en las ISAPREs alcanzó a $10 \%$ de los ingresos de los cotizantes. ${ }^{3}$

El país financia el acceso a la salud de los más pobres en el FONASA con recursos provenientes de impuestos gene-

Cálculo de los autores con información de la Superintendencia de Salud. 
rales. Cerca de 4,5 millones de personas $(35,7 \%$ de beneficiarios del FONASA) están en esta condición; los otros 5,2 millones son cotizantes y sus dependientes (3). En las ISAPREs poco más de 1,4 millones de personas son cotizantes y poco menos de 1,4 millones son sus dependientes (4).

La provisión y el acceso a la atención de salud en el sector privado de las ISAPREs se desenvuelven en un ambiente usual de mercado con acceso a la provisión a través de clínicas y hospitales privados. El sistema público opera poblacional y territorialmente, en un ambiente público organizado en redes jerarquizadas. ${ }^{4}$ Las relaciones entre ambos subsectores son relativamente menores y se producen principalmente a través del sector prestador con la "modalidad de libre elección," ${ }^{5}$ que permite que los cotizantes del FONASA tengan acceso a prestadores privados.

En 2008 el gasto de bolsillo representó un $38 \%$ del gasto total en salud $(5,6)$ convirtiéndose en la principal fuente de financiamiento de la salud, equivalente aproximadamente a $2,8 \%$ del producto interno bruto (PIB) de Chile (7). Recientemente se informó que en 2009 había bajado a $34 \%$, junto con un aumento del gasto total en salud que pasó a representar 8,3\% del PIB $(5,6)$. No obstante, esta cifra del gasto total sigue estando compuesta en $53 \%$ por gasto privado (6). Asimismo, el gasto de bolsillo sigue estando lejos de lo recomendado por la Organización Mundial de la Salud (OMS), que plantea un pago directo máximo de entre $15 \%$ y $20 \%$ del gasto total en salud, dado que uno mayor incrementa significativamente el riesgo de incurrir en gasto catastrófico (2).

FONASA ha contado históricamente con topes de copagos o gratuidad, según el grupo de beneficiarios en que se encuentre el afiliado, ${ }^{6}$ que permiten paliar en parte el problema del gasto de bolsillo

\footnotetext{
4 También existe un sistema especial para las fuerzas armadas que se financia vía impuestos generales y cotizaciones, seguros complementarios privados, las mutuales conforme a la ley 16744 para atención de salud laboral y las cajas de compensación que también gastan en salud.

5 También a través de la ley de urgencia, bonos específicos (AUGE), y el acceso a pensionados de hospitales públicos.

6 Los beneficiarios FONASA se clasifican en grupos: A los más pobres, B, C y D los de mayores ingresos. La atención pública de los grupos A y B es totalmente gratuita, al igual que los adultos mayores, mientras que los grupos $\mathrm{C}$ y $\mathrm{D}$ deben copagar $10 \%$ y $20 \%$, respectivamente, en la atención institucional.
}

en atención de salud y en medicamentos ambulatorios de los sectores más pobres en la atención primaria de salud (APS). Sin embargo, el gasto de bolsillo en Chile es alto al igual que en la mayoría de los países de América Latina y el Caribe (8) y se produce también en los grupos más pobres.

A mediados de 2005, en el contexto de la reforma de salud (Garantías Explícitas en Salud-AUGE) se impulsó la política de protección financiera homogénea, pero solo en el conjunto de problemas de salud con garantías, lo que constituyó un elemento complementario para los beneficiarios del FONASA y totalmente nuevo para los beneficiarios de las ISAPREs. Esta protección financiera consistió en un copago máximo definido como $20 \%$ de un valor fijo del arancel de prestaciones de dichos problemas de salud y un techo de copago anual acumulado para las garantías asociado a la cotización o al ingreso de los afiliados, elemento que fue una novedad para ambos sistemas.

El objetivo de este trabajo es determinar el impacto, las características y los cambios del gasto de bolsillo en salud que individuos y familias debieron enfrentar en Chile en 1997 y en 2007. Se analizan los principales determinantes de este gasto y de la probabilidad de que sean catastróficos, y el efecto de este gasto en la desigualdad de la distribución de los ingresos existente.

\section{MATERIAL Y MÉTODOS}

Se realizó un estudio descriptivo estadístico y econométrico en dos períodos de tiempo para analizar los cambios en el comportamiento de los hogares en Chile utilizando la Encuesta de Presupuestos Familiares (EPF) del Instituto Nacional de Estadísticas (INE) de 1997 y $2007^{7}$ para el Gran Santiago.

El gasto en salud de los hogares recolectado es el gasto de bolsillo en salud o pagos directos (2). ${ }^{8}$ La cotización obliga-

La EPF 1997 fue levantada entre agosto de 1996 y julio de 1997. La EPF 2007 se realizó entre noviembre de 2006 y octubre de 2007. El estudio se limita a comparar hogares del Gran Santiago, dado que la EPF 1997 solo incluyó dicha región.

8 El gasto en salud corresponde a los códigos: 5111 medicamentos; 5211 insumos médicos; 5311 lentes ópticos; 5321 aparatos ortopédicos y terapéuticos; 5411 consulta médica; 5421 intervención médica en operaciones; 5431 honorarios de otros profesionales; 5441 servicio dental; 5451 análisis y servicios de laboratorio y rayos; 5511 servicio de hospitalización; 5521 servicio de emergencia móvil; y 5611 seguro de salud. toria para salud de $7 \%$ no es considerada gasto de bolsillo por la EPF, lo cual es consistente con lo planteado por la OMS (9). Así, el gasto de bolsillo incluye el gasto en consumo final efectivo en servicios, tanto en forma de copagos como pagos completos por atención médica y dental, insumos médicos, aparatos $\mathrm{y}$ equipos auxiliares, atención hospitalaria, farmacológicos y el pago adicional a 7\% de prima voluntaria, dado que este diferencial no es una contribución obligatoria de seguridad social.

El análisis descriptivo estadístico se realiza en base a indicadores del gasto de bolsillo en salud para ambos años y se comparan sus cambios en términos absolutos $^{9}$ relativos a ingresos/gastos totales de los hogares, y su distribución según quintiles de gasto del hogar per cápita.

El análisis principal se efectúa clasificando a los hogares a partir de quintiles de gasto, lo cual es una diferencia significativa con publicaciones anteriores y de otros estudios $(8,10,11)$. De acuerdo a las recomendaciones de la literatura (12), para efectos de estudiar el impacto del gasto de bolsillo sanitario en el bienestar económico del hogar, es conveniente utilizar los quintiles de gasto del hogar per cápita porque este sufre menos variaciones en el tiempo que el ingreso, haciéndolo una mejor medida del bienestar económico del hogar.

El análisis de determinantes del gasto de bolsillo y la probabilidad de incurrir en un gasto catastrófico en salud sigue la metodología propuesta por la OMS $(9,13)$. La capacidad de pago $\left(c_{p}\right)$ es la diferencia entre el gasto total $\left(g_{t}\right)$ y el gasto de subsistencia $\left(g_{s}\right)$ o el gasto en alimentación $\left(g_{a}\right)$ según los siguientes criterios: ${ }^{10}$

\footnotetext{
9 Los valores monetarios están expresados en pesos de abril de 2007.

10 El gasto de subsistencia se define como el gasto mínimo necesario para mantener un nivel de vida básico. Para determinarlo, se calcula la proporción del gasto que se destina a alimentación (excluyendo el gasto en bebidas alcohólicas y tabaco) y luego se divide este gasto por el factor de ajuste por tamaño del hogar para obtener el gasto en alimentación equivalente. El factor de ajuste por tamaño del hogar (escala de equivalencia) se basa en una escala de equivalencia en el consumo, y refleja que aunque el consumo en alimentos aumenta con el número de miembros del hogar, el aumento no es proporcional, i.e.: tamaño equivalente $=$ (tamaño del hogar $)^{0,56}$. El 0,56 fue calculado por un estudio de la OMS (13). Luego se calcula la línea de pobreza como el promedio del gasto en alimentación equivalente de los hogares entre los percentiles 45 y 55 . Finalmente el gasto de subsistencia es la línea de pobreza multiplicada por la escala de equivalencia del hogar. Este representa el gasto mínimo que deberían tener los hogares no pobres.
} 


$$
c_{p}= \begin{cases}g_{s}>g_{a} & g_{t}-g_{a} \\ g_{s} \leq g_{a} & g_{t}-g_{s}\end{cases}
$$

Existe consenso en que el gasto catastrófico en salud se debe medir en base a la capacidad de pago de los hogares. Sin embargo, la proporción de la capacidad de pago que determina cuándo el gasto de bolsillo pasa a ser un gasto empobrecedor depende del contexto que se esté estudiando, con umbrales de entre $20 \%$ y $50 \%(9,14)$. Aquí se tomó un umbral de $30 \%$ para definir el gasto catastrófico, teniendo en cuenta que para el sistema de copagos AUGE la cobertura financiera adicional equivale aproximadamente a $25 \%$ de un ingreso autónomo medio anual por hogar en Chile en 2009, es decir, fue el criterio que se utilizó para dirimir el nivel de gasto aceptable al planificar la garantía financiera.

Para estudiar qué factores explican que los hogares gasten más desde su bolsillo por atención de salud con relación a su capacidad de pago, se utiliza el siguiente modelo Tobit:

$$
\left(\frac{g_{b}}{c_{p}}\right)=\alpha+\beta X_{i}+\mu_{i}
$$

Donde:

$g_{b, i}$ : gasto de bolsillo en salud del hogar $i$ $c_{p, i}$ : capacidad de pago del hogar $i$

$\left(g_{b, i}\right) \quad$ gasto de bolsillo en salud como $\left(\frac{\delta_{b, i}}{c_{p, i}}\right)$ : proporción de la capacidad de pago del hogar $i$

$X_{i}$ : variables independientes del modelo del hogar $i$

$\mu_{i}:$ error

Las variables independientes $\left(X_{i}\right)$ disponibles en las EPF son: número de niños menores de 5 años que viven en el hogar; número de personas mayores a 65 años que viven en el hogar; número de mujeres en edad fértil (entre 15 y 45 años); sexo del jefe del hogar, y años de escolaridad de la pareja del jefe del hogar. También se incluyen variables socioeconómicas, como indicadores de quintil de gasto total del hogar per cápita (omitiendo el primer quintil), indicadores de quintil de ingreso total del hogar per cápita (omitiendo el primer quintil) e indicador de pobreza (definido por la OMS).

Para estudiar qué factores explican la probabilidad de incurrir en gastos catastróficos en salud, se utiliza una regresión logística (9). En este caso la variable dependiente es una variable dicotómica

CUADRO 1. Gasto de bolsillo en salud de los hogares del Gran Santiago, Chile, 1997 y 2007 (en pesos de abril de 2007)

\begin{tabular}{lrrc}
\hline \multicolumn{1}{c}{ Tipo de gasto de bolsillo } & 1997 & \multicolumn{1}{c}{2007} & Diferencia \% \\
\hline Per cápita & 10964 & 15289 & 39,5 \\
Por hogar & 34046 & 41787 & 22,7 \\
Por hogar (como \% del ingreso total) & $5,3 \%$ & $6,9 \%$ & 1,6 \\
Por hogar (como \% del gasto total) & $4,3 \%$ & $4,4 \%$ & 0,1 \\
\hline
\end{tabular}

Fuente: elaboración de los autores con datos de las Encuestas de Presupuestos Familiares (EPF) del Instituto Nacional de Estadísticas (INE), 1997 y 2007.

a Tipo de cambio promedio de abril de 2007 (\$532,3/US\$).

que toma el valor de 1 (uno) si el gasto de bolsillo en salud del hogar es mayor que $30 \%$ de la capacidad de pago del hogar.

$g_{b, i}^{c}=\gamma+\lambda Z_{i}+v_{i}$

Donde:

$g_{b, i}^{c}$ : gasto catastrófico en salud del hogar $i$

$$
\begin{aligned}
& : g_{b, i}^{c}=\left\{\begin{array}{l}
1\left(\frac{g_{b, i}}{c_{p, i}}\right) \geq 30 \% \\
0\left(\frac{g_{b, i}}{c_{p, i}}\right)<30 \%
\end{array}\right. \\
& : g_{b, i}^{c}=\ln \left(\frac{p}{1-p}\right)_{i}
\end{aligned}
$$

$p$ : probabilidad que el hogar $i$ incurra en gasto catastrófico

$Z_{i}$ : variables independientes del hogar $i$ $v_{i}:$ error

En esta especificación se utilizan las mismas variables independientes que en la estimación de los determinantes del gasto de bolsillo en salud.

Finalmente, siguiendo parte de la metodología de otros autores (15) se construyen las curvas de Lorenz y de concentración del gasto de bolsillo en salud y se calculan los índices de Gini y los índices de concentración del gasto de bolsillo. También, se calcula el Gini con los ingresos de la EPF simulando un antes y un después de que los hogares efectúen el gasto de bolsillo en salud, para evaluar cuánto impacta este gasto en la desigualdad de ingreso en Chile.

\section{RESULTADOS}

El gasto del bolsillo promedio mensual en salud que realizan los hogares aumentó en $22,7 \%$, pasando desde un promedio de $\$ 34046$ a $\$ 41787$ mensuales entre 1997 y 2007..$^{11}$ Sin embargo, debido a que el tamaño del hogar dis-

\footnotetext{
11 El tipo de cambio promedio de abril de 2007 fue de 532,3 pesos por US\$.
}

minuyó entre estos dos años, el gasto de bolsillo del hogar per cápita muestra un crecimiento mayor de $39,5 \%$ (cuadro 1 ).

El gasto de bolsillo en salud como porcentaje del ingreso total del hogar aumentó desde 5,3\% a 6,9\% entre 1997 y 2007. Por otro lado, el gasto de bolsillo en salud como porcentaje del gasto total del hogar, básicamente se mantuvo con un $4,3 \%$ en 1997 y 4,4\% en 2007 . Estos fenómenos aparentemente contradictorios se explican porque el gasto total de los hogares creció a la par que el gasto en salud, pero más que el crecimiento del ingreso total del hogar.

Aunque es obvio que en términos absolutos el gasto de bolsillo sea mayor para los hogares pertenecientes a los quintiles de mayores gastos y menor para los hogares de menores gastos, esto también se observa, en alguna medida, en términos relativos. Por ejemplo, el quintil 1 muestra un aumento en el gasto de bolsillo del hogar de $34,6 \%$, pero si se considera el gasto per cápita, que corrige la estimación por el menor tamaño del hogar, el aumento crece a $48,2 \%$. No obstante lo anterior, el aumento también es significativo en los dos quintiles de mayores ingresos (cuadro 2).

La razón entre el gasto en salud del quintil más rico respecto al más pobre pasó desde 30,4 veces en 1997 a 27,5 veces en 2007. Esta disminución de 9,6\% se explica por un aumento en el gasto de los más pobres y un aumento menor del gasto de los más ricos. Sin embargo, esta disminución se atenúa al ver el gasto per cápita, ya que el tamaño del hogar promedio disminuyó entre los 10 años de las encuestas, pero más entre los más pobres. Es así que en el caso del gasto per cápita, la razón entre el quintil más rico y el más pobre disminuyó desde 48,6 veces a 45,8 veces o en $5,8 \%$.

El componente principal del gasto de bolsillo de los hogares es el gasto en medicamentos con un 55\% del gasto total de bolsillo en 2007, siendo proporcional- 
CUADRO 2. Gasto de bolsillo en salud (del hogar y per cápita), como porcentaje del gasto total del Gran Santiago, por quintil de gasto per cápita, Chile 1997 y 2007 (en pesos de abril de 2007)

\begin{tabular}{|c|c|c|c|c|c|c|c|c|c|}
\hline \multirow[b]{2}{*}{ Quintil } & \multicolumn{3}{|c|}{ Gasto del hogar } & \multicolumn{3}{|c|}{ Gasto per cápita } & \multicolumn{3}{|c|}{ Porcentaje del gasto total } \\
\hline & 1997 & 2007 & Cambio \% & 1997 & 2007 & Cambio \% & 1997 & 2007 & Diferencia \\
\hline Quintil 1 (más pobre) & 3487 & 4693 & 34,6 & 774 & 1147 & 48,2 & 2,0 & 2,1 & 0,1 \\
\hline Quintil 2 & 8643 & 10987 & 27,1 & 2210 & 2911 & 31,7 & 3,2 & 3,1 & $-0,1$ \\
\hline Quintil 3 & 18536 & 21601 & 16,5 & 4732 & 6072 & 28,3 & 4,3 & 4,3 & $-0,1$ \\
\hline Quintil 4 & 33619 & 42718 & 27,1 & 9464 & 13766 & 45,5 & 5,1 & 5,6 & 0,5 \\
\hline Quintil 5 (más rico) & 105997 & 128973 & 21,7 & 37660 & 52565 & 39,6 & 6,9 & 7,1 & 0,1 \\
\hline Total & 34046 & 41787 & 22,7 & 10964 & 15289 & 39,5 & 4,3 & 4,4 & 0,1 \\
\hline Razón [Q5/Q1] & 30,4 & 27,5 & $-9,6$ & 48,6 & 45,8 & $-5,8$ & & & \\
\hline
\end{tabular}

Fuente: elaboración de los autores con datos de las Encuestas de Presupuestos Familiares (EPF) del Instituto Nacional de Estadísticas (INE), 1997 y 2007.

a Tipo de cambio promedio de abril de 2007 (\$532,3/US\$).

mente más importante en los quintiles más bajos. Por ejemplo, en el primer quintil fue de $68 \%$ y en el quinto de $47 \%$. Estas cifras se hicieron más críticas respecto de 1997, cuando el gasto medio en medicamentos alcanzaba a 53\% del total de gasto de bolsillo y en el primer quintil a $57 \%$, mientras que en el quinto ascendía a $46 \%$. La incidencia del gasto catastrófico - que supera en $30 \%$ la capacidad de pago del hogar- disminuyó leve pero significativamente en términos estadísticos —de 3,8\% en 1997 a 3,6\% en 2007.

Por otra parte, resulta interesante destacar que son los quintiles de mayor gasto los que se encuentran en mayor probabilidad de caer en gasto catastrófico. De hecho, en 2007, 4,3\% de los hogares del quintil 4 y 5,7\% del quintil 5 tuvieron gastos en salud mayores a $30 \%$ de su gasto total, aumentando de manera significativa respecto de 1997 (4\% y $5,1 \%)$. En cambio, los quintiles más pobres presentaron incidencias de 2,2\% y 2,5\% (quintil 1 y 2, respectivamente), bajando de forma significativa respecto a $1997(3,2 \%$ y $2,8 \%)$.

El cuadro 3 muestra los resultados de las estimaciones de los determinantes del gasto de bolsillo como porcentaje de la capacidad de pago de los hogares según el tipo de variable de ingreso utilizado en la regresión. Así, los resultados que controlan por quintiles de ingreso se presentan en las primeras dos columnas para cada año, seguidos de los resultados que controlan por quintiles de gasto y luego por pobreza.

El número de niños menores de 5 años y el número de personas mayores de 65 años de cada hogar tienen un impacto positivo y significativo en la proporción del gasto de bolsillo en relación con la capacidad de pago de los hogares en ambos años. Sin embargo, en ambos

CUADRO 3. Determinantes del gasto de bolsillo en salud en Gran Santiago, Chile, 1997-2007

\begin{tabular}{|c|c|c|c|c|c|c|}
\hline \multirow[b]{2}{*}{ Variable } & \multicolumn{2}{|c|}{ Quintiles de ingreso } & \multicolumn{2}{|c|}{ Quintiles de gasto } & \multicolumn{2}{|c|}{ Pobreza } \\
\hline & 1997 & 2007 & 1997 & 2007 & 1997 & 2007 \\
\hline No. de niños $<5$ años en el hogar & $\begin{array}{r}0,019 a \\
(0,003)\end{array}$ & $\begin{array}{c}0,014^{a} \\
(0,003)\end{array}$ & $\begin{array}{c}0,022^{a} \\
(0,002)\end{array}$ & $\begin{array}{c}0,019 a \\
(0,003)\end{array}$ & $\begin{array}{c}0,015^{a} \\
(0,002)\end{array}$ & $\begin{array}{r}0,008^{a} \\
(0,003)\end{array}$ \\
\hline No. de personas > 65 años en el hogar & $\begin{array}{r}0,028^{a} \\
(0,003)\end{array}$ & $\begin{array}{r}0,024^{a} \\
(0,004)\end{array}$ & $\begin{array}{r}0,030^{a} \\
(0,004)\end{array}$ & $\begin{array}{r}0,026^{a} \\
(0,005)\end{array}$ & $\begin{array}{c}0,027^{a} \\
(0,003)\end{array}$ & $\begin{array}{r}0,022^{a} \\
(0,005)\end{array}$ \\
\hline No. de mujeres en edad fértil (entre 15-45 años) & $\begin{array}{c}-0,002 \\
(0,002)\end{array}$ & $\begin{array}{c}0,002 \\
(0,002)\end{array}$ & $\begin{array}{c}-0,000 \\
(0,002)\end{array}$ & $\begin{array}{r}0,005^{b} \\
(0,002)\end{array}$ & $\begin{array}{r}-0,006^{a} \\
(0,002)\end{array}$ & $\begin{array}{r}-0,004^{b} \\
(0,002)\end{array}$ \\
\hline Sexo del jefe del hogar (hombre $=1$ ) & $\begin{array}{r}-0,021^{a} \\
(0,006)\end{array}$ & $\begin{array}{r}-0,026^{a} \\
(0,004)\end{array}$ & $\begin{array}{r}-0,016^{a} \\
(0,004)\end{array}$ & $\begin{array}{r}-0,025^{a} \\
(0,004)\end{array}$ & $\begin{array}{r}-0,026^{a} \\
(0,004)\end{array}$ & $\begin{array}{r}-0,031^{a} \\
(0,004)\end{array}$ \\
\hline Edad del jefe del hogar & $\begin{array}{r}0,006^{a} \\
(0,001)\end{array}$ & $\begin{array}{r}0,006^{a} \\
(0,001)\end{array}$ & $\begin{array}{r}0,006^{a} \\
(0,001)\end{array}$ & $\begin{array}{c}0,007^{a} \\
(0,001)\end{array}$ & $\begin{array}{c}0,007^{a} \\
(0,001)\end{array}$ & $\begin{array}{r}0,006^{a} \\
(0,001)\end{array}$ \\
\hline Escolaridad (años) de la pareja del jefe del hogar & $\begin{array}{r}0,003^{a} \\
(0,000)\end{array}$ & $\begin{array}{c}0,003^{a} \\
(0,000)\end{array}$ & $\begin{array}{c}0,002^{a} \\
(0,000)\end{array}$ & $\begin{array}{c}0,002^{a} \\
(0,000)\end{array}$ & $\begin{array}{c}0,003^{a} \\
(0,000)\end{array}$ & $\begin{array}{r}0,003^{a} \\
(0,000)\end{array}$ \\
\hline Quintil 2 & $\begin{array}{r}0,025^{a} \\
(0,006)\end{array}$ & $\begin{array}{c}0,027^{a} \\
(0,006)\end{array}$ & $\begin{array}{r}0,045^{a} \\
(0,006)\end{array}$ & $\begin{array}{r}0,042^{a} \\
(0,006)\end{array}$ & & \\
\hline Quintil 3 & $\begin{array}{c}0,041^{a} \\
(0,006)\end{array}$ & $\begin{array}{c}0,031^{a} \\
(0,007)\end{array}$ & $\begin{array}{c}0,068^{a} \\
(0,007)\end{array}$ & $\begin{array}{r}0,069^{a} \\
(0,006)\end{array}$ & & \\
\hline Quintil 4 & $\begin{array}{r}0,048^{a} \\
(0,006)\end{array}$ & $\begin{array}{r}0,049^{a} \\
(0,006)\end{array}$ & $\begin{array}{r}0,075^{a} \\
(0,005)\end{array}$ & $\begin{array}{r}0,090^{a} \\
(0,006)\end{array}$ & & \\
\hline Quintil 5 & $\begin{array}{c}0,058^{a} \\
(0,005)\end{array}$ & $\begin{array}{c}0,064^{a} \\
(0,006)\end{array}$ & $\begin{array}{c}0,095^{a} \\
(0,006)\end{array}$ & $\begin{array}{c}0,105^{\mathrm{a}} \\
(0,006)\end{array}$ & & \\
\hline Indicador de pobreza (OMS) & & & & & $\begin{array}{r}-0,096^{a} \\
(0,009)\end{array}$ & $\begin{array}{r}-0,108^{a} \\
(0,010)\end{array}$ \\
\hline Constante & $\begin{array}{r}-0,084^{a} \\
(0,007)\end{array}$ & $\begin{array}{r}-0,093^{a} \\
(0,010)\end{array}$ & $\begin{array}{r}-0,113^{a} \\
(0,010)\end{array}$ & $\begin{array}{c}-0,127^{a} \\
(0,011)\end{array}$ & $\begin{array}{r}-0,038^{a} \\
(0,009)\end{array}$ & $\begin{array}{r}-0,047^{a} \\
(0,010)\end{array}$ \\
\hline Observaciones & 8443 & 6480 & 8443 & 6480 & 8443 & 6480 \\
\hline
\end{tabular}

Fuente: elaboración de los autores con datos de las Encuestas de Presupuestos Familiares (EPF) del Instituto Nacional de Estadísticas (INE), 1997 y 2007.

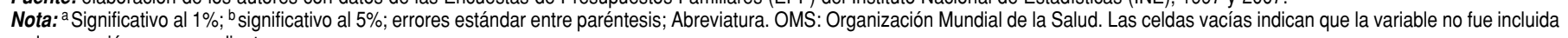
en la regresión correspondiente. 
CUADRO 4. Determinantes del gasto catastrófico en salud en Gran Santiago, Chile, 1997 y 2007

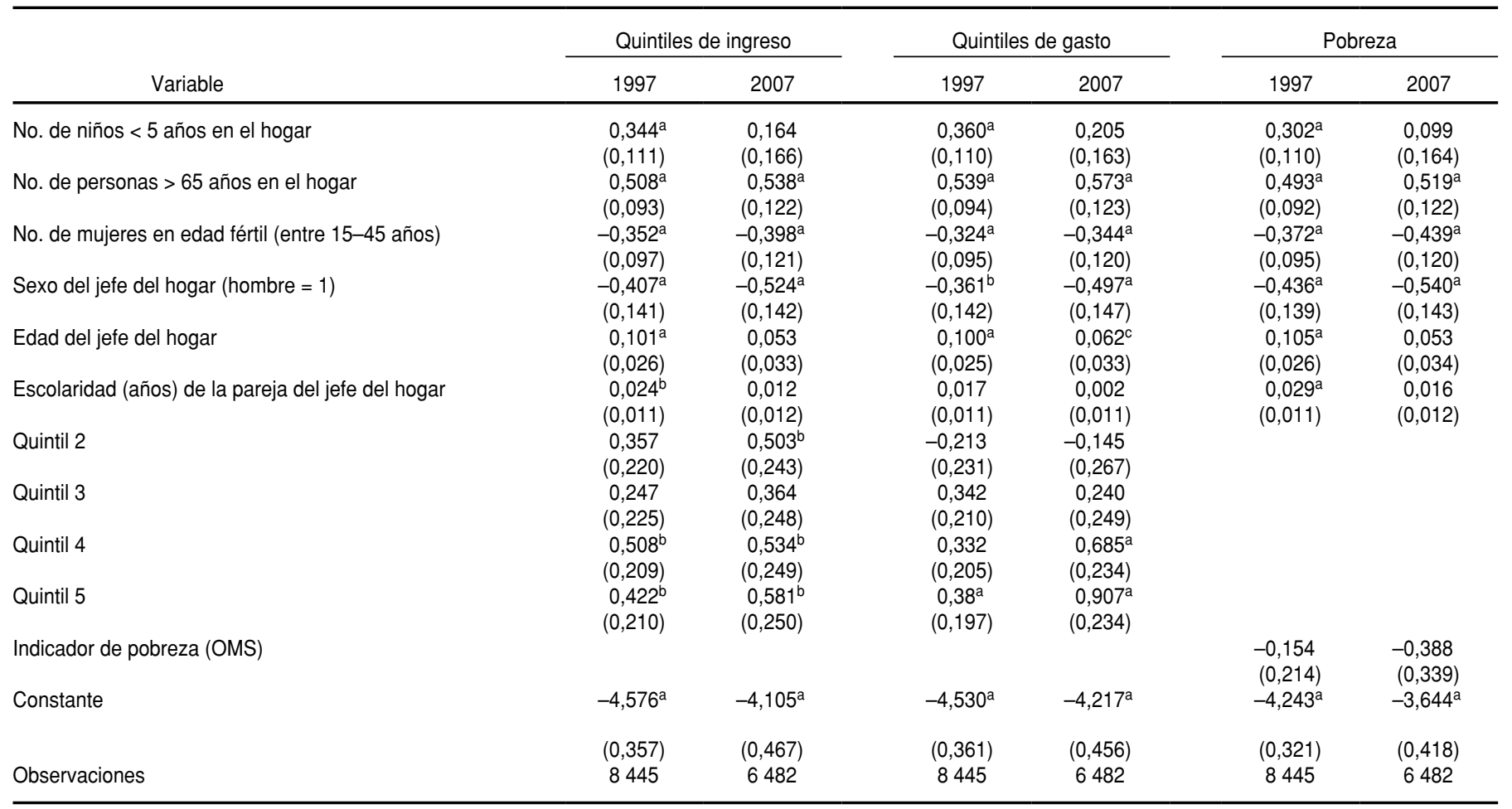

Fuente: elaboración de los autores con datos de las Encuestas de Presupuestos Familiares (EPF) del Instituto Nacional de Estadísticas (INE), 1997 y 2007.

Nota: a significativo al 1\%; ${ }^{\text {b }}$ significativo al $5 \%$; ${ }^{c}$ significativo al $10 \%$; errores estándar robustos entre paréntesis. Abreviatura. OMS: Organización Mundial de la Salud. Las celdas vacías indican que la variable no fue incluida en la regresión correspondiente.

casos el impacto es menor en 2007 que en 1997. Por otro lado, cuando se controla por estado de pobreza, el número de mujeres en edad fértil reduce ligeramente el gasto de bolsillo en salud. No obstante, cuando se controla por quintiles de ingreso no es un determinante significativo, mientras que cuando se controla por quintiles de gasto es significativo y positivo pero muy pequeño en 2007.

Un hogar cuyo jefe es hombre tiene un gasto de bolsillo en salud menor. Una explicación posible a lo anterior es que la definición de jefe de hogar está asociada al rol de proveedor. De esta manera, cuando la mujer es jefa de hogar ella debe salir a trabajar y por lo tanto está principalmente fuera del hogar. Por el contrario, hay fuerte presencia de ella cuando el hombre es el proveedor o jefe de hogar y ella permanece en él.

En el cuadro 4 se pueden ver - presentados de la misma manera que los anteriores- los resultados de las estimaciones de los determinantes de la probabilidad de incurrir en gastos catastróficos. Si bien la presencia de niños menores de 5 años de edad tiene una relación positiva e importante con la probabilidad de incurrir en un gasto de bolsillo catastrófico en 1997, en 2007 esta variable deja de ser un determinante significativo. Por otro lado, la presencia de adultos mayores de 65 años de edad aumenta la probabilidad de gastos catastróficos y la magnitud de su efecto también aumentó entre las dos mediciones. Finalmente, la presencia de mujeres en edad fértil en el hogar disminuye significativamente la probabilidad de gastos catastróficos. Esta relación negativa ha aumentado entre los dos años.

Las mediciones que controlan por nivel de ingreso, sin embargo, muestran que solo para los hogares en los quintiles más altos el nivel de ingreso es una variable importante, lo que puede estar reflejando la mayor disposición a gastar de los quintiles 4 y 5, y que las coberturas de sus planes (usualmente privados) pueden haber disminuido. No obstante, pertenecer al quintil 2 (generalmente FONASA) en 2007 presenta un resultado similar, ya que en ese año se volvió relevante la probabilidad de caer en pobreza en ese grupo. Esto podría ser reflejo de mayores necesidades de gasto en medicamentos ambulatorios no cubiertos en la APS, ni disponibles tampoco en los establecimientos de nivel secundario, junto con un aumento del acceso a estos niveles de atención.

En Chile, las cifras oficiales del coeficiente de Gini del ingreso son de 0,56 en 1996 y 0,53 en 2006 (16). Las EPF arrojan resultados similares, siendo 0,558 y 0,554 en 1997 y 2007, respectivamente, es decir que casi no ha variado. Por otra parte, la concentración del gasto total de los hogares es levemente menor que la del ingreso $(0,54$ y 0,53$)$. Asimismo, como se puede observar en la figura 1, el gasto de bolsillo en salud de los hogares es altamente concentrado y ha crecido con coeficientes de concentración de 0,82 en 1997 y 0,84 en 2007 (figura 1). Por último, al calcular la diferencia entre el coeficiente de Gini con y sin el gasto de bolsillo, se aprecia que los índices de Gini empeoran en 0,012 en 1997 y en 0,015 en 2007, es decir que el gasto de bolsillo en salud es responsable de $2 \%$ (1997) y 2,6\% (2007) de dicho coeficiente.

\section{DISCUSIÓN}

Los resultados indican que entre 1997 y 2007 el gasto de bolsillo en salud ha aumentado fuertemente. Además, la 
FIGURA 1. Ginis y curvas de concentración en base al ingreso o gasto del hogar per cápita. Ginis antes y después de gasto de bolsillo en salud en el Gran Santiago, Chile, 1997 y 2007
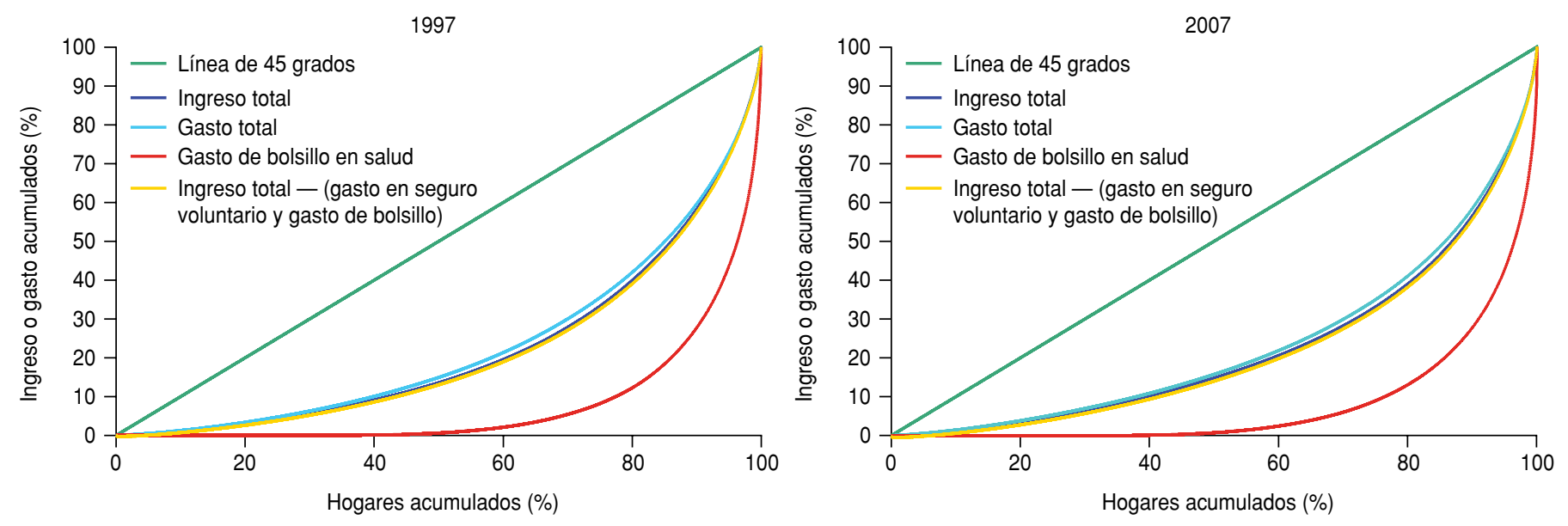

Fuente: elaboración de los autores.

brecha entre la proporción del gasto de bolsillo en salud sobre el gasto total de los hogares más ricos respecto a los más pobres ha disminuido, pero a causa del aumento menor en el estrato más acomodado en comparación con el aumento en los más pobres, lo que contraviene el aspecto positivo de la disminución de la brecha.

La presencia de niños y de personas de tercera edad tiene una relación positiva con el gasto de bolsillo, y la tendencia es que el efecto sea menor. Esto puede deberse al mejoramiento de las coberturas financieras en programas muy masivos que apuntan a estos grupos poblacionales, como el de infecciones respiratorias agudas (IRA) en niños — que se incorporó al AUGE en 2005- y el de enfermedades respiratorias en adultos mayores (ERA).

Por otro lado, el número de mujeres en edad fértil se reduce ligeramente y en algunos casos no es significativo para el gasto de bolsillo en salud, lo que podría ser una contradicción ya que los costos esperados en salud para este grupo son relativamente más altos. Esto podría ser reflejo del continuo nexo con el sistema de salud que en general las mujeres tienen cuando están en edad de fértil y durante los embarazos (por las constantes visitas a la red de atención primaria de salud), lo que a su vez podría tener efectos positivos sobre el acceso a beneficios y a la salud del conjunto de miembros del hogar.

La probabilidad de incurrir en gasto catastrófico entre ambos años dismi- nuyó levemente, lo que podría deberse a una mejor protección financiera o un aumento en la capacidad de pago de los hogares. Entre los determinantes, se observa un aumento en la presencia de adultos mayores de 65 años, lo que podría estar mostrando la permanente falta de protección financiera de los hogares con adultos mayores, que no logra ser revertida a pesar de los programas implementados. Por ejemplo, el AUGE en su primer año incluyó solo cataratas y la artrosis de cadera severa que requiere prótesis como patologías especialmente dedicadas a adultos mayores. ${ }^{12}$

A diferencia de lo que ocurre en 1997, en 2007 la presencia de niños ya no parece influir en el gasto de bolsillo. Parece entonces razonable asociar en cierta medida algunos de los resultados obtenidos con la eficacia de algunos programas de salud, como por ejemplo de aquellos que son amplios y universales y enfocados hacia los niños. Por ejemplo, entre 1997 y 2007 hubo un salto importante en el programa de atención a las IRA, que pasó de 350 salas de tratamiento en 1997 a cerca de 802 en 2007, es decir 2,3 veces más en este último año ${ }^{13}$ (17). Más todavía, a partir de julio de 2005 se sumaron otras patologías dirigidas a niños con

\footnotetext{
12 Otras patologías dentro de los 25 problemas de salud que incorporaban a adultos mayores, como la diabetes y la hipertensión arterial, ya venían funcionando con anterioridad y pueden no haber generado mayores cambios en el gasto de bolsillo.

13 En 2007 se distribuyeron más de 3,3 millones de inhaladores (p. ej. salbutamol, budesonida, salm+flutic, ipratropid y salmeterol).
}

garantías AUGE, en este caso con un impacto probable a nivel hospitalario, como por ejemplo las cardiopatías congénitas operables, fisura labiopalatina, cáncer infantil y prematurez (18). En julio de 2006 se incorporaron algunas patologías asociadas a adultos mayores que pueden no haber alcanzado a reflejar un efecto en la encuesta, tales como vicios de refracción en adulto y desprendimiento de retina (18).

Adicionalmente, el número de mujeres en edad fértil demostró no tener un impacto significativo en la incidencia del gasto catastrófico, lo que puede deberse a las mismas razones ya señaladas en el análisis de determinantes del gasto, esto es, a efectos en la economía del hogar y cercanía a dispositivos de salud primaria de las mujeres. Por último, el gasto de bolsillo es muy concentrado y promueve inequidades. A pesar de que los datos generales de distribución del ingreso mejoran levemente, el gasto de bolsillo en salud aumenta su desigualdad y su impacto en la desigualdad general entre las dos mediciones.

Las políticas de protección financiera deben fomentar la equidad, reducir la desigualdad de acceso a la salud, impedir el empobrecimiento de las familias por razones de salud y fortalecer las políticas para alcanzar la universalidad (2). Si la política de protección financiera del sistema no se fortalece para propender estos resultados, a futuro solo se podrá esperar un aumento de la desprotección financiera y de la desigualdad en su distribución. 


\section{REFERENCIAS}

1. Organización Mundial de la Salud. Informe sobre la salud en el mundo 2000 - Mejorar el desempeño de los sistemas de salud. Ginebra, Suiza: OMS; 2000.

2. Organización Mundial de la Salud. Informe sobre la salud en el mundo 2010 - Financiación de los sistemas de salud: el camino hacia la cobertura universal. Ginebra, Suiza: OMS; 2010.

3. Gobierno de Chile, Fondo Nacional de Salud. Estadísticas institucionales: Demografía. FONASA 2011. Disponible en: http://www. fonasa.cl/wps/wcm/connect/internet/ sa-general/informacion+corporativa/ estadisticas+institucionales/estadisticas+ institucionales Acceso el 19 de febrero de 2012.

4. Gobierno de Chile, Superintendencia de salud. Cartera de beneficiarios ISAPRE, 2010. Disponible en: http://www.supersalud.gob. $\mathrm{cl} /$ documentacion/569/w3-article-6900.html Acceso el 19 de febrero de 2012.

5. Organisation for Economic Co-operation and Development. Health at a Glance 2011: OECD Indicators. París, Francia: OECD; 2011.

6. World Health Organization. Estimates for country NHA data. Geneva: WHO; 2011. Disponible en: http://www.who.int/nha/country/ chl/en/ Acceso el 19 de febrero de 2012.
7. Cid C. Financiamiento agregado de la salud en Chile: 2008. Cuadernos Médico Sociales. 2011;51(3):143-50.

8. Perticara M. Incidencia de los gastos de bolsillo en salud en siete países latinoamericanos. Serie Políticas Sociales 141. Santiago, Chile: CEPAL; 2008

9. Xu K. Distribución del gasto en salud y gastos catastróficos. Discussion paper No. 2. Ginebra, Suiza: WHO; 2005.

10. Agacino R. La cuenta satélite de salud, CSS, serie 2003-2007. Reseña y principales resultados. Economía y Salud. Boletín del Departamento de Economía de Salud. Ministerio de Salud (MINSAL). 2009;2(1):7-14.

11. Cid C. La persistencia del gasto de bolsillo de los hogares en salud. Cuadernos Médico Sociales. 2009;49(1):44-8.

12. Rogers JM, Gray MB. CE Data: Quintiles of Income versus Quintiles of Outlays. Monthly Labor Review. 1994;118(8).

13. Xu K, Evans DB, Kawabata K, Zeramdini R, Klavus J, Murray CJ. Household catastrophic health expenditure: a multicountry analysis. Lancet. 2003;362(9378):111-7.

14. Torres AC, Knaul FM. Determinantes del gasto de bolsillo en salud e implicaciones para el aseguramiento universal en México: 1992-2000. En: Knaul FM, Nigenda G, eds.
Caleidoscopio de la Salud. De la investigación a las políticas y de las políticas a la acción. México: Fundación Mexicana para la Salud; 2003. Pp. 209-25.

15. O'Donnell O, van Doorslaer E, Wagstaff A, Lindelow M. Analyzing health equity using household survey data analyzing: A guide to techniques and their implementation. Washington, D.C.: World Bank; 2008.

16. Gobierno de Chile, Ministerio de Desarrollo Social. Encuesta de Caracterización SocioEconómica Nacional (CASEN). Disponible en: http://www.ministeriodesarrollosocial.gob. $\mathrm{cl} /$ casen/ Acceso el 19 de febrero de 2012.

17. Gobierno de Chile, Ministerio de Salud. Avances en Salud 2007. Disponible en: http:// www.minsal.gob.cl/portal/url/item/6fef2d0 115888b76e04001011f016550.pdf Acceso el 19 de febrero de 2012.

18. Inostroza M, Riesco X. La reforma de salud en Chile a cuatro años de su implementación. En: Sánchez Rodríguez H, ed. La salud del bicentenario, Chile 2011-2020: desafíos y propuestas. Santiago, Chile: Instituto Salud y Futuro. Universidad Andrés Bello; 2011. Pp. 67-92.

Manuscrito recibido el 22 de junio de 2011. Aceptado para publicación, tras revisión, el 17 de enero de 2012.

ABSTRACT Objective. Determine the impact, characteristics, and changes in out-of-pocket health spending of households in Chile in 1997 and 2007.

\section{Out-of-pocket health spending: the case of Chile, 1997 and 2007}

Methods. A descriptive econometric study was conducted based on household surveys with cross-sectional information on spending in two years-1997 and 2007-for Greater Santiago. The evolution of indicators of per capita household spending by quintile was reviewed. The method proposed by the World Health Organization was used for econometric analysis of the determinants of out-of-pocket spending and catastrophic spending. The Gini indices were also calculated to analyze equity.

Results. Out-of-pocket health spending in the households of Greater Santiago increased significantly (39.5\% per capita). The ratio of health spending in quintile 5 compared to quintile 1 decreased, but the increase in spending was less in quintile 5 . In 2007, out-of-pocket spending was still determined by the presence of risk groups: under 5 years and, although to a lesser extent, over 65 years of age. Catastrophic spending decreased slightly and the presence of older adults continues to increase this risk. The presence of women of childbearing age tends to minimize out-of-pocket spending.

Conclusions. Out-of-pocket health spending in the households is high and has increased. It continues to have a significant influence on inequality. The effects of out-of-pocket spending containment programs such as AUGE are evident, but new financial protection policies that address the problem are needed.

Key words Financing, health; health expenditures; financing, personal; equity in health; Chile. 